\title{
De la intencionalidad de la conciencia \\ a la mundanidad del mundo. \\ Husserl como antecedente inmediato de \\ Heidegger
}

\author{
Edgar Rodríguez Aguilar \\ Academia de Ciencia Política \\ Universidad Autónoma de la Ciudad de México \\ edgarrodrigueza@gmail.com
}

\section{Introducción}

El presente trabajo tiene el objetivo de presentar, de manera general, el camino que ofreció a Heidegger la posibilidad de tematizar la mundanidad del mundo.

No creemos que, en general, se pueda hablar de «profetas» y «Mesías» en filosofía y, en el caso particular, diferimos de la posibilidad de que aquellos puedan ser, por muy cercanas que parezcan sus posturas, Lukács y Ortega y este último, Heidegger ${ }^{1}$. Sin embargo, la revolución filosófica que representa la obra de Heidegger no nos parece que sea producto sólo de la espontaneidad creativa. Todo lo contrario: creemos que la posibilidad de desarrollar una filosofía enraizada en la vida que dé cuenta de la manera en que el hombre es, originariamente, en el mundo - ser-ahí como ser-enel-mundo-, desde un acercamiento fenomenológico, fue posible en Heidegger gracias al desarrollo que sus predecesores Brentano, pero sobre todo Husserl, llevaron a cabo en torno al problema de la conciencia y su correlato, el mundo. Cabe señalar que este no es el único camino desde el que Heidegger llega a la tematización del Dasein, pues según algunos biógrafos es aproximadamente en

${ }^{1}$ Esto a diferencia de: Francisco Gil Villegas, Los profetas y el mesías. Lukács y Ortega como precursores de Heidegger en el zeitgeist de la modernidad (1900-1929), México: FCE-CM 1998. 
1921 que el autor de la Selva Negra «redescubre» el horizonte de los griegos a la luz de Hölderlin, Schelling y Nietzsche.

Es este camino el que intentamos rescatar aquí para ver cómo ha sido posible la revolución filosófica que representa la obra de Heidegger al construir una verdadera ontología ${ }^{2}$ desde la cual sea posible hacer justicia al legado fenomenológico de «ir a las cosas mismas», de tematizar la vida fáctica en la que inmediatamente nos encontramos.

Para esto intentamos seguir como hilo conductor la crítica de Husserl a la clásica escisión sujeto-objeto a partir de la intencionalidad de la conciencia - rescatada por él- propuesta por Brentano en su consideración de los «fenómenos psíquicos» (primer paso). Sobre esta base, considerada sobre todo a partir de las Investigaciones lógicas (IL), Quinta y Sexta, en las cuales el autor describe la relación entre la conciencia intencional y el objeto mentado de manera vertical, atendemos a la horizontalidad que en Ideas relativas a una fenomenología pura y una filosofía fenomenológica (Ideas) adopta Husserl y que permite a Heidegger hablar de la mundanidad del mundo. Estamos concientes de la importancia que tiene la crítica de Heidegger a la fenomenología reflexiva de Husserl y el papel que ésta juega en la transformación hermenéutica de la fenomenología. Sin embargo, hemos decidido seguir sólo el camino mencionado.

Este camino nos parece pertinente dado que desde las primeras dos décadas del siglo XX, Heidegger fue llevado al problema central del que se ocuparía su obra, tanto previa como posterior a El ser y el tiempo: el sentido del ser³. Según el propio Heidegger, la disertación de Brentano de 1862 titulada Del múltiple significado

2 Podría parecer muy paradójico hablar de una «verdadera ontología». Sin embargo, usamos la expresión para hacer notar el hecho de que según Heidegger en la historia de la metafísica lejos de haberse constituido una ontología se hizo una onto-teología. Ésta se explica, en buena medida, a partir de la división entre los mundos inteligible y sensible, y la obvia preeminencia del primero sobre el segundo en la historia de la filosofía.

${ }^{3}$ Como es claro, no coincidimos con las perspectivas según las cuales la obra de Heidegger está fragmentada y cada fragmento trata problemas filosóficos distintos. Creemos que desde lo que algunos han llamado la obra temprana de Heidegger hasta el pensar del Ereignis, el autor trata el sentido del ser, sólo que la manera de tematizarlo es distinta en el transcurrir de su vida y obra. 
del ente según Aristóteles fue lo que lo llevó al problema que lo ocuparía toda su vida: ¿cuál es el sentido del ser si el ente puede ser dicho desde significados múltiples? ${ }^{4}$

Al mismo tiempo que tanto la perspectiva de Brentano como su innovadora interpretación de Aristóteles lo llevaban a este problema ${ }^{5}$, su lectura de las Investigaciones lógicas, sobre todo la Sexta, y en especial la distinción que Husserl hace en ella entre «intuición sensible»e «intuición categorial», lo convencieron de tratarlo desde las posibilidades de la fenomenología, pues según él, «Lo que las investigaciones fenomenológicas habían encontrado de manera nueva como sustentación del pensar se probaba como el rasgo fundamental del pensamiento griego, si es que no de la filosofía en cuanto tal $»^{6}$.

\section{Primer paso: La crítica de Husserl a la escisión sujeto-objeto}

Aunque finalmente la fenomenología husserliana cayera no sólo en la tradición de la «filosofía tradicional», como la llama Heidegger, sino, concretamente, en la tradición del idealismo cartesiano a causa de la búsqueda de conceptualizar una conciencia pura, así como de formular una fenomenología trascendental, Husserl es capaz de romper con la clásica escisión sujeto-objeto, sobre la cual opera no sólo la historia de la metafísica, sino la teoría moderna del conocimiento ${ }^{7}$.

4 Cf. Martin Heidegger, «Mi camino en la fenomenología», en: Tiempo y ser, Madrid: Tecnos 2001, p. 95.

${ }^{5}$ En el texto antes citado Heidegger hace énfasis en la importancia de Aristóteles en la conformación de la fenomenología. Algunos intérpretes también la han encontrado e incluso sistematizado, hasta el punto de que, por ejemplo, según Franco Volpi El ser y el tiempo no representa más que, de manera extraordinaria, una traducción de la Ética Nicomáquea. Algunas consideraciones respecto a las tesis de Volpi se pueden ver en: Alejandro VIGo, «Síntesis y diaíresis: un motivo aristotélico en las teorías fenomenológicas del juicio de Husserl y Heidegger», en: Héctor Zagal y Alberto Fonseca (comps.), Aristóteles y aristotélicos, México: Publicaciones Cruz O. 2002.

${ }^{6}$ Heidegger, Mi camino en..., p. 100.

7 Esto es de suma importancia, pues es con referencia a esta teoría del conocimiento, 
A partir de la separación y presunta inconmensurabilidad entre la subjetividad cognoscente y los objetos del mundo, la filosofía se dedicó a buscar los medios necesarios para aminorar su distanciamiento o, en el caso extremo de Hegel, lograr su reconciliación. Sin embargo, ya Husserl se dio cuenta del hecho no sólo de que si es posible hablar de una relación entre partes inconmensurables es porque esta presunta inconmensurabilidad no está del todo acabada, sino - esto es lo decisivo - de que auténticamente no hay separación alguna. No es posible hablar - aunque, como ya mencionamos, posiblemente Husserl recaiga en esto- de conciencia o subjetividad puras, separadas de cualquier objeto, así como no es posible, tampoco, hablar de objetos puros, emancipados de cualquier relación, ya sea con una subjetividad $-I L-$ o con otros objetos - Ideas - . Ya desde la Primera Investigación Lógica Husserl deja claro el sentido de objetividad. Ésta no hace referencia a algún mundo nouménico de objetos en sí que difieran de su carácter fenoménico, de aquello que representan para el sujeto. La objetividad está en el cumplimiento significativo, a partir de la correlación entre el acto de dar sentido y el acto de cumplir sentido, mediados por la aparición intuitiva de los objetos mentados, ya sean reales o fantasiosos.

Husserl se adhiere críticamente a la distinción de Brentano entre fenómenos psíquicos y físicos. De la conceptualización de Brentano de la conciencia en el sentido de acto psíquico, Husserl retoma dos de las seis definiciones ofrecidas por aquél. La primera remite a la relación y referencia de la conciencia a un contenido específico. Es ésta la esencia de los actos o fenómenos psíquicos, de acuerdo con la cual: «En la percepción es percibido algo; en la representación imaginativa es representado imaginativamente algo; en el enunciado es enunciado algo; en el amor es amado algo;

cuyos pilares son los pensamientos cartesiano y kantiano, que Heidegger habla de que la única época que ha sido capaz de crearse una imagen del mundo a partir de la radical separación de un sujeto que se lo representa y un objeto - el mundo- representado, ha sido la moderna. Cf. Martin Heidegger, «La época de la imagen del mundo», en: Caminos de bosque, Madrid: Alianza Editorial 2000. 
en el odio es odiado algo; en el apetito es apetecido algo, etc.» ${ }^{8}$. Con esto es clara la ruptura de la escisión conciencia-objeto y el «regreso» ${ }^{9}$ a una correspondencia más originaria en la que toda conciencia lo es de algo, se trata de una intencionalidad que es dirigida hacia algo, pues ¿cómo pensar en los fenómenos del escuchar, ver, sentir, tocar, admirar, sin su necesaria correlación con aquello que es o puede ser escuchado, visto, sentido, tocado, admirado? ¿Cómo pueden ser pensados estos fenómenos de la conciencia sin el contenido necesario al que ella ha de dirigirse?

La segunda definición de Brentano retomada por Husserl se refiere al hecho de que los fenómenos psíquicos, o son representaciones o se erigen sobre ellas. Para que un juicio, deseo, temor o cualquier otro acto se dé, es necesario que sea representado aquello que se juzga, se desea, se teme. Esto es muy importante porque llevará al autor, en la Sexta Investigación Lógica, a hablar de actos fundamentados que sólo son posibles sobre la base de actos primitivos fundamentantes; distinción que, a su vez, le permitirá desarrollar la diferencia entre las intuiciones sensible y categorial.

8 Edmund Husserl, Investigaciones lógicas 2, Madrid: Alianza 2002, Quinta Investigación, p. 491.

9 Cabe destacar la importancia de la palabra «regreso», puesto que dentro de la búsqueda de los medios más adecuados para establecer los puentes ente la conciencia subjetiva y los objetos del mundo - ya sea que se trate de las ideas, la res cogitans, las categorías del entendimiento o cualquier otro-, algunos pensadores del siglo XIX hablaban de la necesidad de acudir nuevamente a los legados kantiano, fichteano, hegeliano, etc. Sin embargo, para Husserl el pensamiento es libre, no está comprometido con nadie, y la filosofía, en tanto empresa estrictamente científica y objetiva, no puede ser ciega seguidora de pensamientos $\mathrm{u}$ hombres, sino que ha de ser fiel sólo «a las cosas mismas». Husserl enuncia esta crítica a los prejuicios al decir: «no fue un llamado afortunado el 'Volvamos a Kant', que irremisiblemente trajo consigo al poco tiempo los equisonantes llamados 'Volvamos a Fichte', 'Volvamos a Hegel', a Fries, a Schopenhauer. El llamado legítimo reza de nuevo: A las cosas mismas como espíritus libres, con un interés puramente teórico», en: Edmund HuSSERL, «La relación del fenomenólogo con la historia de la filosofía», en: Antonio Zirión (comp.), Actualidad de Husserl, México: Alianza 1989, pp. 16-17. La crítica de la fenomenología, no sólo de Husserl sino de Heidegger, a los prejuicios como obstáculos que impiden una mirada libre y directa de los fenómenos, se puede ver en: Ramón Rodríguez, La transformación hermenéutica de la fenomenología. Una interpretación de la obra temprana de Heidegger, Madrid: Tecnos 1997, pp. 71-73. Así pues, el primer paso para plantear un regreso a los orígenes es la máxima fenomenológica husserliana de un regreso «a las cosas mismas» (Zu den Sachen Selbst!). 
Pero de esto hablaremos más adelante. Por lo pronto queremos dejar en claro que en la Quinta Investigación Lógica Husserl hace hincapié en que a pesar de que no puede haber diferencia alguna ente el contenido vivido o conciente y la vivencia, pues, como explica Ramón Rodríguez, «Lo sentido, por ejemplo, no es otra cosa que la sensación» ${ }^{10}$, sí hay una distinción entre el objeto y el acto. El objeto no forma parte del contenido de la vivencia, puede existir o no; lo único importante es el acto, pues en la conciencia no se encuentra el objeto, sino sólo el contenido de la conciencia intencional: «Si existe el objeto intencional, nada cambia desde el punto de vista fenomenológico. Lo dado es para la conciencia exactamente igual, exista el objeto representado, o sea fingido o incluso contrasentido» ${ }^{11}$. Esto hace posible el hecho de que distintas menciones, llevadas a cabo incluso desde aproximaciones opuestas, puedan realizarse acerca de uno y el mismo objeto. Sólo a partir de la «independencia» de objeto y acto es posible hablar, siguiendo un ejemplo de Husserl, del «vencedor de Jena y el vencido de Waterloo» ${ }^{12}$ haciendo referencia al mismo objeto.

Como mencionamos, la descripción que hace Husserl de la manera en que opera la conciencia en las $I L$ es desde la intencionalidad vertical. Es hasta la definición de «intuición categorial» donde comienzan a vislumbrarse las posibilidades de que los objetos se den, es gracias a ella que comienza a romperse la verticalidad de la intencionalidad para dar paso a la horizontalidad. Como muy bien lo expone Ramón Rodríguez, las consideraciones de Husserl en Ideas, a diferencia de las $I L$, le permiten incluir al objeto intencional en el análisis fenomenológico. Según él, esto fue posible sólo a partir de dejar de considerar la región psíquica de las vivencias como un lugar privilegiado frente a la región física; es decir, al considerar que la conciencia intencional «no se agota en los componentes reales de los actos, sino que se extiende hasta el objeto mismo» ${ }^{13}$.

10 Rodríguez, La transformación hermenéutica..., p. 39.

11 Husserl, Investigaciones lógicas 2, Quinta Investigación, p. 495.

12 Edmund Husserl, Investigaciones lógicas 1, Madrid: Alianza 2001, Primera Investigación, p. 249.

13 Rodríguez, La transformación hermenéutica..., p. 48. 
Para los fines del presente texto esto es muy significativo, pues nos acerca cada vez más a la concepción heideggeriana de la mundanidad gracias a que deja de privilegiarse la capacidad racional y cognoscente del sujeto en el acto de conocimiento; deja de enfatizarse al Dasein como sujeto que observa a los entes del mundo que tiene al frente para concebir, más bien, una correlación entre ambos, una fundición de ambos en el mundo ${ }^{14}$. No debemos entender que es la inclusión del objeto lo que marca el paso de la intencionalidad vertical a la horizontal, sino, al contrario, que su inclusión se da cuando es posible que aparezca en un entorno significativo, ya no de manera «independiente» como en la mención directa, sino relacionado en una estructuración compleja de sentido. Y esto, como mencionamos, sólo es posible a partir de la «intuición categorial».

Retomemos ahora la definición de Brentano que dice que todo fenómeno psíquico, o es una representación o tiene a la base representaciones. Husserl llega a una interpretación del término representación como «todo acto en el cual algo se hace para nosotros objetivo en cierto sentido estricto, a modo de las percepciones y de las intuiciones paralelas, que aprehenden de un solo golpe o mientan en un solo rayo de mención su objeto, o a modo de los actos-sujetos unimembres en los enunciados categóricos, o de los actos de simple suposición, que funcionan como primeros miembros en los actos de enunciación hipotética, etc.» ${ }^{15}$. Con esta definición Husserl intenta postular la existencia de actos simples a partir de cuya función fundamentadora es posible la construcción de otros más complejos. Esos actos, que llama «objetivantes», constituyen el amplio género que abarca a todas las vivencias intencionales. Así, la definición de Brentano es transformada por Husserl en lo siguiente: «toda vivencia intencional, o es un acto objetivante, o tiene un acto objetivante por $\left\langle\right.$ base $>\gg{ }^{16}$.

${ }^{14} \mathrm{El}$ autor citado sigue esta línea de interpretación, pues menciona: «la idea de la intencionalidad como correlación inseparable vivencia-objeto y la <superación> de la región psíquica, resultados ambos del abandono de la posición básica de las Investigaciones lógicas, marca justamente el núcleo de la concepción heideggeriana de la fenomenología», en: RoDRíGUEZ, La transformación hermenéutica..., p. 49.

15 HusserL, Investigaciones lógicas 2, Quinta Investigación, p. 555.

16 Husserl, Investigaciones lógicas 2, Quinta Investigación, p. 578. 
Uno de los puntos más importantes en la aprehensión de los objetos del mundo que lleva a cabo la conciencia es la unidad dinámica de identificación. En ésta, que sólo es posible sobre la base de los actos objetivantes, entran en juego la intención de dar significado y la intención de cumplir el significado mentado en aquélla, de cuya coincidencia resulta el cumplimiento significativo. Este cumplimiento se puede dar en mayor o menor medida dependiendo de la manera como el objeto aparezca. Así, la mayor plenitud está no en la imaginación, pues en ella el cumplimiento se da por medio de la semejanza con la cosa, sino en la percepción, pues a pesar de que se aproxima a los objetos por escorzos, sólo en ella aparece la cosa tal como es en sí misma ${ }^{17}$.

Para los fines del hilo conductor que guía nuestra interpretación, resulta de central importancia el hecho de que el cumplimiento significativo, que está atravesado por la intuición correspondiente a lo mentado en la intención significativa y con el cual se da la unidad de identificación, no se da por ninguna reflexión comparativa, sino que sólo es posible en tanto es vivido ${ }^{18}$. La única limitación que esto representa para una tematización plena de la vida fáctica es la rigidez con la que la direccionalidad de la conciencia es vivida; el cumplimiento significativo no se da de manera reflexiva, sino que se vive ${ }^{19}$, pero la vivencia se da única $y$

17 Encontramos aquí uno de los logros más importantes de la fenomenología, posibilitado por Brentano, pero presentado de manera más acabada por Husserl y Heidegger: la necesaria correspondencia entre la conciencia y los objetos a los que se dirige y la imposibilidad de una conciencia pura trascendental separada de cualquier objeto. A pesar de la recaída de Husserl en la tradición cartesiana, aquí es muy clara la oposición a la dualidad kantiana noúmeno-fenómeno, pues el autor menciona: «es de observar que el objeto, tal como es en sí -en sí en el único sentido pertinente y razonable aquí, sentido que el cumplimiento de la intención de la percepción realizaría-, no es totalmente distinto de cómo la percepción lo realiza, aunque sólo sea de un modo imperfecto. Está implícito, por decirlo así, en el sentido propio de la percepción, el ser la aparición del objeto mismo», en: HusserL, Investigaciones lógicas 2, Sexta Investigación, pp. 638-639.

18 Husserl, Investigaciones lógicas 2, Sexta Investigación, pp. 623-624.

${ }^{19}$ Cabe señalar que este vivir no reflexivo que llevará a Husserl al concepto de «actitud natural», no sólo es compartido por Heidegger, sino que es lo que le permite hablar de lo originario, aquello de lo cual la fenomenología en tanto ciencia - originaria - ha de ocuparse. Lo problemático no es aquello de lo que se ocupa la fenomenología, pues se trata, tanto en Husserl como en Heidegger, de la «actitud natural», del carácter «originario» de las 
exclusivamente respecto del objeto mentado, cuyo cumplimiento significativo es posible gracias a la intuición correspondiente. La unidad de significación es descrita desde una intencionalidad estrictamente vertical en referencia sólo al objeto mentado al que en el momento específico del que se trate está dirigida la conciencia. Como mencionamos, esta verticalidad es superada en Ideas gracias a la adopción de un punto de vista horizontal respecto a la intencionalidad. Pero esto sólo es posible a partir de la «intuición categorial», desarrollada en la última de las $I L$.

Después de los detallados análisis que en las IL hace Husserl de la manera como la conciencia se relaciona con los objetos específicos a los que se dirige, y una vez que ha llegado a la exposición de que la verdad sólo se da en la vivencia de la evidencia, es decir, en la plena coincidencia entre el objeto mentado y el que aparece en la intuición correspondiente a él, el autor se pregunta si el cumplimiento significativo se da de la misma manera en los momentos simples de la significación que en los categoriales. Una vez que ha probado que de la manera descrita se da la unidad de identificación en los actos nominales simples, surge el problema de si sucede lo mismo ya no al hablar de lo rojo, el libro, el tintero, la mesa o, incluso, ese libro, ese tintero o esta mesa, sino de enunciados más complejos de la forma el libro que está sobre la mesa, o el carro rojo es más pequeño que el azul, pero más veloz.

En estos casos la significación no puede confundirse con la per-

vivencias; se trata de la vida fáctica. Lo que resulta problemático es el acceso a ella. Esto es lo que hace la distinción entre las fenomenologías de ambos autores, lo que a pesar del camino compartido y el objetivo común de «ir a las cosas mismas», los llevará a posturas radicalmente disímiles. Pues, para Husserl la única manera de explicitar las vivencias de la actitud natural es poniéndolas en suspenso, objetivándolas, poniéndolas al frente para describirlas. La crítica de Heidegger es que si realmente se quiere ir «a las cosas mismas», el proceder fenomenológico debe ser igual de originario que la vida fáctica que va a ser tematizada y explicitada. Si la mirada científica objetiva la vivencia de la que se ocupa, si vive su relación con ella de manera teórica a partir de la relación sujeto-objeto, entonces tiende a describirla de manera teórica, a partir de la relación sujeto-objeto. Con esto, lejos de ir a la cosa misma - las vivencias en la actitud natural - , tal como ella se presenta, se da un acercamiento modificado. Por eso el acceso no puede ser reflexivo, sino hermenéutico; la vida sólo puede ser tematizada desde la vida misma, y lo que hace la epojé al suspender la vivencia en la actitud natural para objetivarla no es más que un des-vivir. 
cepción. En los enunciados simples la significación propia «mantiene una simple relación de coincidencia con la percepción» ${ }^{20}, \mathrm{y}$ esto gracias a que hay un contenido real específico, es decir, un correlato objetivo de lo mentado cuyo cumplimiento pleno es posible a través de la intuición correspondiente. Pero en el caso de las significaciones formadas, aquellas cuya complejidad reside en ser estructuras relacionantes, hay algo más, algo que no tiene un correlato objetivo, un contenido real específico al que corresponda una intuición más o menos adecuada. Es este «plus» que no encuentra cumplimiento en la percepción directa puesto que no puede ser aprehensible acudiendo a ninguna intuición sensible, el que delimita el terreno de lo categorial.

Los complejos de objetos relacionados, de cuya estructuración resulta la significación, no pueden aprehenderse directamente a través de la intuición simple o sensible por la falta de un correlato real, objetivo, que pueda ser intuido. Sin embargo, ese «plus» que escapa a toda percepción y vuelve compleja la situación está formado de notas simples que sí pueden cumplirse intuitivamente. Por lo tanto, si bien el cumplimiento no se da por la percepción, sino por la significación, sigue siendo necesaria la percepción de las notas simples a partir de las cuales se hace posible la significación de la estructuración compleja. Es decir, la percepción y la intuición correspondiente sirven para fundir los actos unificantes o relacionantes que estructuran la enunciación compleja. Así, según Husserl, «Si juntamos estos actos fundados, o más bien, estas formas de acto, con sus actos fundamentantes y comprendemos bajo el título de acto fundado los actos complejos enteros que nacen mediante esta fundamentación formal, podemos decir: en el supuesto de la posibilidad que acabamos de señalar, se restablece el paralelismo, sólo que ya no es un paralelismo entre las intenciones significativas de las expresiones y las meras percepciones correspondientes a ellas, sino entre las intenciones significativas y aquellos actos que están fundados en las percepciones» ${ }^{21}$.

${ }^{20}$ HusserL, Investigaciones lógicas 2, Sexta Investigación, p. 695.

${ }^{21}$ HusserL, Investigaciones lógicas 2, Sexta Investigación, p. 695. 
El cumplimiento significativo en las formas categoriales es posible porque se da una intuición o percepción categoriales no de los objetos aislados a los que corresponde un correlato real directo, sino de nuevas objetividades. Éstas son constituidas por actos relacionantes o unificantes que están fundamentados por otros: por los actos simples cuyo cumplimiento está en la intuición o percepción de sus objetos reales correspondientes. Husserl menciona:

Al verificarse los nuevos actos de la conjunción, de la disyunción, de la aprehensión individual determinada e indeterminada (esto-algo), de la generalización, del conocer simple, relacionante y unificante, no surgen unas vivencias subjetivas cualesquiera, ni tampoco unos actos en general enlazados con los primitivos, sino unos actos que constituyen nuevas objetividades, como hemos dicho; surgen actos en los cuales aparece algo como real y como dado ello mismo, pero de tal suerte que este algo, tal como aparece aquí, todavía no estaba dado ni podía estarlo en los actos fundamentantes solos. Más por otra parte la nueva objetividad se funda en la antigua; tiene referencia objetiva a la que aparece en los actos fundamentantes. Su modo de aparecer está determinado esencialmente por esta referencia. Trátese aquí de una esfera de objetividades que sólo pueden aparecer <ellas mismas> en actos de tal suerte fundados ${ }^{22}$.

Los objetos no son otros, sino los mismos, pero relacionados y estructurados en objetividades complejas. Husserl no hace explícitamente la distinción entre objeto y objetividad en este sentido. Sin embargo, queda expuesta al hablar de objetos más amplios configurados a partir de los más simples. Es aquí donde las cosas ya pueden aparecer dentro de un determinado horizonte no sólo espacial, sino temporal, pues es de acuerdo a la distinta forma de configuración de los objetos más amplios - objetividades - que tanto ellos como los objetos más simples cobran significatividad. Esto es claro cuando Husserl dice que «es menester que sea destacado un miembro como miembro capital y que sea considerado a la vez que se fijen los restantes miembros correlativos; siendo de advertir que también estos tienen, notoriamente, que destacarse, y

22 Husserl, Investigaciones lógicas 2, Sexta Investigación, p. 705. 
que aquella determinación cambia según la especial índole de la unidad dominante» ${ }^{23}$.

Como podemos ver, con la «intuición categorial» Husserl puede dejar la rigidez de la intencionalidad vertical en la que sólo se hace presente el objeto mentado, para dirigirse hacia la intencionalidad que abarca una objetividad más compleja en la que son destacados varios objetos que entran en el campo de percepción más amplio; dirección que, como veremos a continuación, lo lleva a una horizontalidad en la que cobran importancia, incluso, aquellos objetos que, aunque no están presentes en el campo de percepción, están en -y constituyen - el mundo.

III. Segundo paso: De la verticalidad de la mirada intencional a la horizontalidad del mundo circundante

Las ideas anteriores, referentes a las $I L$, muestran el primer paso hacia una filosofía enraizada en la vida y en el mundo a partir de la superación de la clásica escisión que la metafísica, y claramente su correlato en la teoría moderna del conocimiento, llevó a cabo entre un sujeto racional cognoscente y un objeto susceptible de ser conocido, como realidades independientes.

Sin embargo, dada la importancia que en ellas tiene el hacer una descripción de las vivencias de la conciencia intencional en relación con el objeto mentado, los análisis parten de una relación vertical entre estos dos elementos de la aprehensión (conciencia intencional y objeto mentado). Es decir, Husserl en las IL intenta describir cómo es que la conciencia entra en relación y aprehende los objetos del mundo, y esto sólo lo puede hacer describiendo la pura relación entre ellos. La importancia de este primer paso reside «sólo» en el hecho de haber roto con el punto de vista desde el cual lo imperante era establecer el puente más adecuado para conciliar dos mundos que en la historia de la metafísica aparecían hasta cierto punto como inconmensurables: el subjetivo y el objetivo.

${ }^{23}$ HusserL, Investigaciones lógicas 2, Sexta Investigación, p. 711. 
Pero el camino husserliano hacia una tematización plena de la vida fáctica no se queda en la verticalidad de las descripciones llevadas a cabo en las $I L$, las cuales desde el punto del vista del autor son estrictamente fenomenológicas en sentido puro por tratarse de indagaciones puramente inmanentes de esencias y por hablar no de leyes psicológicas, sino de «posibilidades y necesidades puras, que son inherentes a cualquier forma del cogito puro, por ser contenidos reales e intencionales o por sus conexiones posibles a priori con otras tales formas en una conexión de conciencia idealiter posible en general» ${ }^{24}$. Tras la crítica a la escisión sujeto-objeto a partir de las consideraciones acerca de la intencionalidad de la conciencia, el segundo paso fue proporcionar un punto de vista horizontal de las vivencias, el cual, desde nuestra lectura, es decisivo en la conformación del fenómeno del mundo y la significatividad de éste por plexos de sentido, conceptos desarrollados más tarde en la fenomenología hermenéutica heideggeriana.

Gracias a la horizontalidad a la que Husserl alude en Ideas, la conciencia es situada en un mundo que la rodea. No es que en las IL la conciencia no lo estuviera, pero, como mencionamos, dada la importancia descriptiva que en ellas tiene la íntima relación entre la direccionalidad de la conciencia y el objeto aprehendido - mas no contenido - en ella, el mundo que circunda a la relación no había cobrado aún la importancia debida, aparecía como algo borroso y sólo cobraban claridad los objetos que caían dentro de la intuición, los objetos individuales a los que era dirigida la mirada o cualquier otra forma de la percepción sensible.

Ahora ya no sólo se tiene conciencia de los objetos específicos

${ }^{24}$ Husserl, Investigaciones lógicas 2, Sexta Investigación, p. 772. Cabe destacar que al mismo tiempo que la crítica del conocimiento de Husserl posibilita romper con la dicotomía sujeto-objeto, propia de la teoría del conocimiento, cae, en su afán por construir una ciencia estricta que establezca las posibilidades a priori del conocimiento sobre las cuales tendría que estar sustentada cualquier otra ciencia, desde la perspectiva de Heidegger en la tradición del idealismo cartesiano. Parece que desde consideraciones críticas muy distintas respecto de la filosofía, tanto Husserl como Nietzsche ocupan un lugar similar dentro de la historia de ésta, sobre todo respecto de la consolidación del pensamiento heideggeriano. Pues, también desde la perspectiva de éste, Nietzsche es quien ha podido dar el primer paso en la destrucción de la metafísica siendo el último gran metafísico. 
que se encuentran bajo el dominio de la mirada o la escucha, sino que, según Husserl: «Tengo conciencia de un mundo extendido sin fin en el espacio y que viene y ha venido sin fin en el tiempo» ${ }^{25}$. Es con la conciencia de este mundo extendido que Husserl considera la vivencia de un modo más originario, pues claramente la conciencia, aunque nunca es pura en el sentido de una subjetividad trascendente separada de los objetos, ya no es presentada desde la vivencia fundamentante de los actos, sino ubicada en - experienciando - un mundo que la desborda, la abraza, la circunda, en el cual, como más tarde dirá Heidegger, el ser-ahí se encuentra.

Situado en un mundo y a través de los sentidos están las cosas corpóreas, según Husserl, «para mí simplemente ahí, 'ahí delante'»². Pero este estar «ahí delante» no tiene el mismo significado aquí que en las $I L$. En éstas Husserl considera que una de las formas más adecuadas del cumplimiento significativo es la percepción, pues en ella el objeto se hace presente, aparece frente a nosotros tal como es él mismo. Al parecer, por la naturaleza de las investigaciones llevadas a cabo en dicho texto, el objeto aparece frente a la conciencia que se dirige a él y desaparece cuando la direccionalidad cambia. En la perspectiva horizontal y más originaria de las vivencias, el sentido del estar «ahí delante» no es el de hacer frente, sino el de lo inmediato, el de lo dado de ese mundo extendido que circunda a la conciencia, en el cual ésta se encuentra ya fácticamente ${ }^{27}$, pues las cosas del mundo - animadas e inanimadas - están ahí delante «lo mismo si fijo la atención especialmente en ellas, ocupándome en considerarlas, pensarlas, sentirlas, que-

25 Edmund Husserl, Ideas relativas a una fenomenología pura y una filosofía fenomenológica, México: FCE 1949, p. 64.

${ }^{26}$ Husserl, Ideas relativas a..., p. 64 .

27 De ahí que para la fenomenología, a diferencia de las ciencias positivas que deben construir, primero, su objeto de investigación y, segundo, los métodos más idóneos para abordarlo, el «objeto» de estudio no requiere ningún tipo de construcción, pues se trata de la vida, en la cual inmediatamente ya se está. Como mencionamos, lo problemático, en todo caso, es la forma de acceder a ella. Al respecto, cf. se Ángel XoLocotzi, Der Umgang als "Zugang". Der hermeneutisch-phänomenologische Zuzang zum faktischen Leben in den 'frühen Freiburger Vorlesungen' Martin Heidegges im Hinblick auf seine Absetzung von der transzendentalen Phänomenologie Edmund Husserls, Berlin: Duncker und Humbolt 2002, trad. Ángel Xolocotzi, parágrafos 14 y 15, y Rodríguez, La transformación hermenéutica... 
rerlas $o$ no» ${ }^{28}$. La conciencia no deja de ser intencional en el camino de la postura vertical a la horizontal, pero la direccionalidad respecto de los objetos aparece como menos rígida y, sólo en este sentido, menos unidireccional.

A diferencia de la importancia que en la percepción juega la presencia, tanto física como intuitiva, de los objetos apercibidos, sobre todo porque, como se mencionó, es en ella donde se encuentran los niveles más altos de cumplimiento de los actos impletivos, en la estancia dentro de la extensión horizontal del mundo, la conciencia está en relación «indirecta» con los objetos: «no es necesario que ni ellos - los demás hombres - ni los demás objetos, se encuentren justamente en mi campo de percepción. Para mí están ahí los objetos reales, como objetos determinados, más o menos conocidos, a una con los actualmente percibidos, sin que ellos mismos estén percibidos, ni siquiera intuitivamente presentes» ${ }^{29}$.

Con la adopción de la horizontalidad de la conciencia Husserl puede debilitar la región conceptual y reflexiva del acto de conocimiento para acudir a una dimensión más originaria de éste, la cual en Ideas es apresada bajo el nombre de «actitud natural». Dice Husserl:

Puedo dejar peregrinar mi atención desde la mesa de escribir en que acabo de fijarla justamente con la vista, pasando por las partes no vistas del cuarto que están a mi espalda, hasta el balcón, el jardín, los niños que juegan en el cenador, etc., hasta todos los objetos de los cuales justamente 'sé' que están acá o allá en el contorno inmediato que entra en mi campo de conciencia - saber que no tiene nada de un pensar conceptual y que únicamente al variar la dirección de la atención, y aún entonces sólo parcialmente, y las más de las veces muy imperfectamente, se convierte en un claro intuir ${ }^{30}$.

Ya desde las $I L$ se dejaba ver el hecho de la existencia de un trasfondo borroso, del cual emergen, clarificándose, los objetos que caen dentro de la direccionalidad de la conciencia, dentro de

\footnotetext{
28 HusserL, Ideas relativas a..., p. 64. Las cursivas son nuestras.

${ }^{29}$ Husserl, Ideas relativas a..., p. 64 .

${ }^{30}$ Husserl, Ideas relativas a..., pp. 64-65.
} 
la fijación ya sea de la mirada, el olfato, el oído o cualquier otra forma de percepción sensible. Sin embargo, el énfasis lo puso Husserl en la descripción de los procesos de direccionalidad y fijación de la conciencia en las vivencias intencionales. Sólo hasta aquí - Ideas, ese trasfondo borroso en el que inmediatamente se encuentra la conciencia ya de facto, cobra importancia.

En la horizontalidad involucrada en la cita anterior se puede ver además un rasgo que llegará hasta El ser y el tiempo: la relación que los objetos guardan entre sí y que constituyen la totalidad de la significatividad del mundo. La importancia de esto reside en que no sólo están relacionados en una totalidad significativa los objetos que entran en el campo de la percepción, como ocurría en las IL; el horizonte, tomando el ejemplo de Husserl, no sólo es la mesa de escribir, el balcón, el jardín, los niños, sino que, en tanto que se trata de un «horizonte oscuramente conciente de realidad indeterminada» ${ }^{31}$, las relaciones que si bien no están dentro del campo de la percepción, no dejan de estar ahí, en el mundo, pues: «una niebla vacía, de oscura indeterminación, se puebla de intuitivas posibilidades o presuntividades, y sólo se diseña la 'forma' del mundo justamente en cuanto 'mundo'. El contorno indeterminado es, por lo demás, infinito. El nebuloso horizonte, nunca plenamente determinable, está necesariamente ahí» ${ }^{32}$.

El cambio de sentido que adquiere el estar «ahí delante» en el paso de la verticalidad de la conciencia, en la que se dirige a los objetos, a la horizontalidad en la que las múltiples relaciones que constituyen el mundo la circundan inmediatamente, nos parece centralmente significativo porque permite a Heidegger establecer el problema del ser-en-el-mundo. Dado que el mundo ahora está «ahí delante» en un sentido menos literal, en la vivencia natural la conciencia no lo enfrenta como algo extraño que se le presenta, sino que es parte de él, «no está para mí - dice Husserl - ahí como un mero mundo de cosas, sino, en la misma forma inmediata, como un mero mundo de valores y de bienes, un mundo práctico» ${ }^{33}$.

\footnotetext{
31 Husserl, Ideas relativas a..., p. 65.

32 Husserl, Ideas relativas a..., p. 65.

33 Husserl, Ideas relativas a..., p. 66.
} 
Así como en otro muy citado ejemplo Heidegger describe la vivencia de la cátedra no como una serie de percepciones independientes de cuya suma resultaría su significatividad, sino como inmediatamente le es dada la significatividad de la cátedra en la vivencia misma, en la cual, dice el autor, «no veo la cátedra de un golpe, y no la veo aislada; veo el púlpito como demasiado alto para mí. Veo un libro que está encima, lo veo inmediatamente como estorbándome (un libro, y no un cierto número de hojas, guarnecido con pastas negras), veo la cátedra en una orientación, en una iluminación, en un trasfondo» ${ }^{34}$; así, según Husserl, en la actitud natural uno se encuentra con las cosas ejecutándolas de alguna manera, «encontrándolas bellas y feas, gratas e ingratas, agraciadas y desgraciadas, agradables y desagradables, etc. En forma inmediata hay ahí cosas que son objetos de uso, la 'mesa' con sus libros, el 'vaso', el 'florero', el 'piano', etc. (...) Lo mismo vale, naturalmente, así como para las meras cosas, también para los hombres y animales de mi contorno. Son ellos mis 'amigos' o 'enemigos', mis 'servidores' o 'jefes', 'extraños o 'pacientes', etc.» ${ }^{35}$.

Al situar a la conciencia y la intencionalidad en un mundo que las circunda, un mundo en el que se encuentran y desde el cual, como dirá Heidegger, hacen frente a los objetos que están en él, Husserl permite a Heidegger romper completamente la relación teorética sujeto-objeto a partir de una correlación originaria entre el ser-ahí y el mundo, y, con esto, ir «a las cosas mismas».

\section{El ser-ahí como ser en el mundo}

Como acabamos de mencionar, el haber situado Husserl a la conciencia en un mundo que la circunda, le permitió a Heidegger hablar de una situación originaria en la que se encuentra el ser-ahí - término con el que el autor hace referencia al hombre - como un ser-en-el-mundo. Sin embargo, para esto Heidegger necesita

34 Cit. en: Zur Bestimmung der Philosophie en: Rodríguez, La transformación hermenéutica..., p. 24.

35 Husserl, Ideas relativas a..., p. 66. 
liberarse no sólo, como hizo Husserl, de la preeminencia de un lugar privilegiado de la conciencia, como era la región psíquica, sino de la preeminencia que en Husserl sigue teniendo la conciencia a pesar de estar en la horizontalidad del mundo.

No se trata ya de una conciencia que se dirige específicamente a un objeto del mundo para apercibirlo, clarificándose y regresando al trasfondo borroso una vez que la mirada o cualquier otra forma de la percepción sensible cambia de dirección. Pero tampoco se trata de que la conciencia está en una relación indirecta con los objetos que la circundan. En la caracterización del ser-ahí como ser-en-el-mundo no hay actos que la conciencia cumpla con mayor o menor plenitud a través de la intuición de los objetos. Pero tampoco hay una relación indirecta entre conciencia y mundo como si éste estuviera siempre ya no borroso, sino oculto.

Heidegger no habla de la diferencia entre acto y objeto, ni de conciencia intencional y objeto mentado. Establece una diferencia entre el ser-ahí y el resto de los entes que no tienen la forma del ser-ahí. Pero no lo hace para describir cómo se relacionan, ni mucho menos para analizar cómo el ser-ahí aprehende a los entes que no son de su misma forma. Lo hace para mostrar el fenómeno de la mundanidad ${ }^{36} \mathrm{y}$ con esto comprender las determinaciones constitutivas del ser-ahí para construir su ontología fundamental.

¿Por qué para llevar a cabo una analítica existenciaria del ser del ser-ahí el autor tiene que hablar de los fenómenos del mundo y la mundanidad?

Básicamente porque lo que establece la diferencia entre el serahí y el resto de los entes es que mientras que aquel es un ser-enel-mundo, éstos sólo están dentro del mundo.

Antes de continuar cabe destacar que el ser-en-el-mundo representa siempre una unidad ontológica, mas no un todo estructurado a partir del ensamblaje de sus partes. Sin embargo, esto no

36 Cabe destacar que es prácticamente imposible caracterizar o conceptualizar el fenómeno del mundo. Heidegger menciona: «Y cuando se plantea la cuestión del 'mundo', ¿qué mundo se mienta? Ni éste ni aquél, sino la mundanidad del mundo en general». Martin Heidegger, El ser y el tiempo, México: FCE 2000, §14, p. 77. Más bien se trata de mostrar a qué fenómeno hace alusión el término mundanidad del mundo. 
impide hablar de los fenómenos, como el mundo, que constituyen la unidad.

Mundo no hace referencia a un espacio físico como puede ser el planeta Tierra, en el cual se encuentran tanto el ser-ahí como el resto de los entes, sino a un fenómeno. Este fenómeno es una determinación ontológica sólo del ser-ahí, no del resto de los entes. Sin embargo, su caracterización no puede prescindir de éstos y de su ser. El mundo, que lleva al término de lo «mundano», mienta «una forma de ser del ser-ahí y nunca una de los entes 'ante los ojos' ‘en' el mundo» ${ }^{37}$. Ser-ahí es fundamentalmente ser-en-el-mundo. Propiamente sólo él tiene mundo, pues éste es una estructura que lo constituye ontológicamente. No hay, en definitiva, ser-ahí que no sea ser-en-el-mundo. A diferencia de esto, el resto de los entes, que carecen estructuralmente de él, son seres intramundanos que sólo están ubicados y estructurados - por el ser-ahí- dentro del mundo.

Es sólo sobre la base del imprescindible ser-en-el-mundo del ser-ahí, que podemos llegar al fenómeno de la mundanidad ${ }^{38}$. Ahora bien, el autor menciona: «Por ser inherente al 'ser ahí' el 'ser en el mundo', es su 'ser relativamente al mundo' en esencia 'curarse de'» ${ }^{39}$. Pero ¿qué es este «curarse de» que ha de llevarnos al fenómeno de la mundanidad?

El ser-ahí a diferencia, por ejemplo, del «yo trascendental» kantiano es inmediatamente ya en el mundo; es arrojado a un mundo pre-establecido con el que se las tiene que ver, y no a un mundo que es constituido por él mediante las categorías del entendimiento. En este mundo el ser-ahí realiza actividades y pensamientos, lleva a cabo una vida siempre práctica en la que se ocupa de algo o deja de ocuparse de ello para hacerlo de otra cosa. El «curarse de», en este sentido, se entiende como el llevar a cabo cotidiano que el ser-ahí en tanto ser-en-el-mundo realiza. En este «curarse de» el

37 Heidegger, El ser y..., §14, p. 78.

38 Según Heidegger, «el no ver la estructura del 'ser ahí', que es 'ser en el mundo' trae consigo un pasar por alto el fenómeno de la mundanidad», en: HeIdegger, El ser y..., §14, p. 78.

${ }^{39}$ Heidegger, El ser y..., §12, p. 70. 
ser-ahí se encuentra con los entes que le hacen frente en el mundo que lo circunda; «anda» en el mundo con los entes intramundanos que le hacen frente. Pero este «hacer frente» de los entes intramundanos no remite al sentido que Husserl utiliza en las $I L$, como objetos que son percibidos e intuidos cognoscitivamente para dar cumplimiento significativo, ni al que hace alusión en Ideas como ese mundo que está ahí delante, ya entre en mi actual campo de percepción o no, sino al «curarse de» manipulando entes. En palabras de Heidegger: «La forma inmediata del 'andar' no es (...) el conocimiento no más que perceptivo, sino el 'curarse de' que manipula ${ }^{40}$.

De esta manera, los entes que no tienen la forma de ser del serahí cobran sentido inmediatamente por la utilidad que el ser-ahí les da en su andar cotidiano «curándose de». Su ser está inmediatamente determinado por «servir para algo» en el «curarse de». Como mencionamos, estos entes carecen de mundo, en el sentido estructural en que él es para el ser-ahí; sólo están dentro de él ocupando un lugar físico que en última instancia es determinado por las necesidades del ser-ahí en su «ocuparse de» cotidiano.

Pero, como dijimos, el ser-ahí no llega al mundo a fundamentar ni a establecer cuál ha de ser el sentido que los entes han de tener. Entonces, ¿cómo es posible que el ser-ahí sepa o conozca cómo han de ser utilizados los entes en su cotidiano «curarse de» si él no funda los sentidos de manera cognoscitiva, es decir, si no los conoce previamente para después ejecutarlos?

En cierta medida el mundo está constituido por una totalidad de entes intramundanos que están relacionados bajo una totalidad de útiles en la que un útil remite siempre a otros útiles. Heidegger menciona:

El útil respondiendo a su 'ser útil', 'es' siempre por la adscripción a otro útil: palillero, pluma, tinta, papel, carpeta, mesa, lámpara, mobiliario, ventanas, puerta, cuarto. Estas 'cosas' jamás se muestran inmediatamente por sí, para luego llegar como una suma de cosas reales un cuarto. Lo que hace frente inmediatamente, si bien no aprehendi-

${ }^{40}$ Heidegger, El ser y..., §15, p. 80. 
do temáticamente, es el cuarto, pero tampoco éste como lo 'entre las cuatro paredes', en un sentido espacial, geométrico, sino como útil para habitar o habitación. Partiendo de ésta se muestra el 'arreglo' de la misma y en él el útil 'singular' del caso. Antes que este último es en cada caso ya al descubierto una totalidad de útiles ${ }^{41}$.

El fenómeno del mundo nos remite a aquella estructura compleja que desde nuestra perspectiva Heidegger puede tematizar a partir de la «intuición categorial» desarrollada por Husserl. Sólo que en este caso no se trata de actos objetivantes que constituyen, sobre la base de actos más simples, objetos más amplios que como tales han de ser intuidos para cumplir los actos de dar significado. Se trata, más bien, de relacionarse con el ser de los entes intramundanos que hacen frente al ser-ahí a partir de utilizarlo, de tomarlo como «ser a la mano». Pero en la cita anterior el autor menciona que antes de que aparezca el útil singular del caso, es decir, determinado útil que se hace necesario en un momento específico de la cotidianidad para que el ser-ahí se ocupe en lo que en ese momento y contexto es el caso, para que pueda «curarse de», se encuentra ya al descubierto una totalidad de entes. Para poder utilizar un ente específico es necesaria la base referencial de la totalidad de entes intramundanos interconectados.

Originariamente no se trata de que el ser-ahí no conoce el mundo en el que se encuentra y éste le hace frente de manera hostil, ni de que el ser-ahí dude si el mundo que tiene al frente es verdadero o no. Por el contrario, «El mundo es en todo lo 'a la mano' siempre ya 'ahí'. El mundo es previamente descubierto, si bien atemáticamente, con todo lo que le hace frente» ${ }^{42}$.

El mundo es siempre ya conocido no en sentido epistémico, teórico o cognoscitivo, sino en el sentido de que inmediatamente le es familiar al ser-ahí. El ser-ahí está en una familiaridad con el mundo de acuerdo con la cual no funda los sentidos de utilidad que los entes que le hacen frente en su cotidiano «curarse de» tienen, sino que los realiza, y esto gracias a que al ser-ahí le es in-

${ }^{41}$ Heidegger, El ser y..., §15, p. 82.

42 Heidegger, El ser y..., §18, p. 97. 
herente una comprensión de los entes y del ser. Es gracias a esta comprensión que puede ser «descubierto» ese fondo que funge como base referencial desde la cual los entes cobran sentido y que el ser-ahí puede manipular siempre en vistas de un "para qué». Ese fondo no puede ser más que la totalidad de la significatividad que conforma el mundo, en la cual están los plexos de significación desde los cuales cobran sentido los entes intramundanos. Heidegger menciona al respecto:

Aquello dentro de lo cual el 'ser ahí' se comprende previamente en el modo del referirse, es el 'aquello sobre el fondo de lo cual' del previo permitir que hagan frente entes. El 'aquello dentro de lo cual' del comprender refiriéndose, en cuanto 'aquello sobre el fondo de lo cual' del permitir que hagan frente entes en la forma de ser de la conformidad, es el fenómeno del mundo. Y la estructura de aquello sobre el fondo de lo cual se refiere el 'ser ahí' es lo que constituye la mundanidad del mundo ${ }^{43}$.

El fondo que sustenta la significatividad del mundo debe ser «descubierto» para que el ser-ahí pueda «curarse de» en su andar cotidiano; la única manera de «descubrirlo» es gracias a la precomprensión que el ser-ahí tiene tanto del ser como de los entes; la precomprensión la tiene el ser-ahí inmediatamente en tanto que ha sido arrojado a un mundo estructurado significativamente, es decir, le es inherente en tanto ser-en-el-mundo; entonces, en el mundo ese fondo es descubierto por el ser-ahí inmediatamente en su cotidiano «curarse de».

\section{Consideraciones finales}

Una de las venas más importantes en la conformación de la fenomenología de Heidegger es, como intentó mostrarse, el camino crítico que viene de Brentano y Husserl, y el objetivo de «ir a las cosas mismas». 
El hilo conductor que seguimos aquí para intentar dilucidar cómo fue posible que Heidegger llegara a la tematización del modo más originario de ser del ser-ahí como ser-en-el-mundo, fue a partir de su cercanía con el pensamiento de los autores mencionados. Sin embargo, con esto sólo atendimos a una parte en la conformación de su pensamiento. Queda otra que es de suma importancia. Se trata no del seguimiento que hace de Husserl, sino de su distanciamiento. Éste tiene que ver más que con los temas tratados, con el procedimiento fenomenológico; con el hacer a un lado el imperativo de una ciencia estricta e intentar llevar a cabo una ciencia originaria si se quiere cumplir la máxima fenomenológica. Aunque esta discusión no la pudimos abordar, salvo en una nota a pie de página ${ }^{44}$, queremos finalizar comentando que el proceder fenomenológico heideggeriano, radicalmente distinto a la epojé husserliana, muestra el carácter originario desde el que la vida misma se da. Así, por ejemplo, la manera en que Heidegger puede tematizar el fenómeno de la mundanidad, en el cual, como vimos, el ser-ahí se encuentra inmediatamente manipulando entes, es sólo desde ciertas «rupturas» que se dan en el cotidiano «curarse de», como son la sorpresa, la impertinencia y la insistencia, gracias a las cuales es posible que el mundo se muestre a través de destellos. 


\section{RESUMEN}

El presente trabajo tiene el objetivo de exponer, de manera general, el camino que ofreció a Heidegger la posibilidad de tematizar la mundanidad del mundo. El autor defiende que la revolución filosófica que representa la obra de Heidegger no es producto sólo de la espontaneidad creativa. Todo lo contrario: la posibilidad de desarrollar una filosofía enraizada en la vida que dé cuenta de la manera en que el hombre es, desde un acercamiento fenomenológico, fue posible gracias al desarrollo que Brentano y Husserl llevaron a cabo en torno al problema de la conciencia y su correlato, el mundo.

Palabras clave: Heidegger, Husserl, mundanidad, intencionalidad.

\section{ABSTRACT}

The present paper exposes, in general, the way that offered Heidegger the possibility to explain the worldliness of the world. The author defends that the revolution that represents Heidegger's work is not only a product of creative spontaneity. On the contrary: the possibility to develop a philosophy that comprehend the way of being of human being, in a phenomenological sense, it is possible only hence the developments of Husserl and Brentano on the topics of conscience and world.

Key words: Heidegger, Husserl, worldliness, intentionality. 